\title{
Topological Invariant and Quantum Spin Models from Magnetic $\pi$ Fluxes in Correlated Topological Insulators
}

\author{
F. F. Assaad, M. Bercx, and M. Hohenadler \\ Institut für Theoretische Physik und Astrophysik, Universität Würzburg, Am Hubland, 97074 Würzburg, Germany
}

(Received 23 April 2012; revised manuscript received 5 October 2012; published 26 February 2013)

\begin{abstract}
The adiabatic insertion of a $\pi$ flux into a quantum spin Hall insulator gives rise to localized spin and charge fluxon states. We demonstrate that $\pi$ fluxes can be used in exact quantum Monte Carlo simulations to identify a correlated $Z_{2}$ topological insulator using the example of the Kane-Mele-Hubbard model. In the presence of repulsive interactions, a $\pi$ flux gives rise to a Kramers doublet of spin-fluxon states with a Curie-law signature in the magnetic susceptibility. Electronic correlations also provide a bosonic mode of magnetic excitons with tunable energy that act as exchange particles and mediate a dynamical interaction of adjustable range and strength between spin fluxons. $\pi$ fluxes can therefore be used to build models of interacting spins. This idea is applied to a three-spin ring and to one-dimensional spin chains. Because of the freedom to create almost arbitrary spin lattices, correlated topological insulators with $\pi$ fluxes represent a novel kind of quantum simulator, potentially useful for numerical simulations and experiments.
\end{abstract}

DOI: 10.1103/PhysRevX.3.011015

Subject Areas: Strongly Correlated Materials, Topological Insulators

\section{INTRODUCTION}

A topological insulator represents a novel state of matter characterized by a special band structure that can result, e.g., from strong spin-orbit interaction [1,2]. In two dimensions, this state is called a quantum spin Hall insulator and has deep connections with the quantum Hall effect, including the coexistence of a bulk band gap and metallic edge states, the absence of symmetry breaking, and the possibility of a mathematical classification [3,4]. Importantly, because of the absence of a magnetic field, the quantum spin Hall insulator preserves time-reversal symmetry, which provides protection against interactions and disorder [5-7]. The quantum spin Hall insulator has been realized in HgTe quantum wells $[8,9]$.

Correlated topological insulators with strong electronelectron interactions are the focus of current research [10]. Intriguing concepts include electron fractionalization in the presence of time-reversal symmetry, [11-14] spin liquids [14-16], and topological Mott insulators [17,18]. Remarkably, some of the theoretical models can be studied using exact numerical methods. A central problem in this context is the question of how to detect a topological state directly from bulk properties, for example, in cases where the bulk-boundary correspondence breaks down. Experimentally, this issue also arises in the absence of sharp edges in proposed cold-atom realizations as a result of the trapping potential $[19,20]$. The classification in terms of a $Z_{2}$ Chern-Simons index relies on Bloch wave

Published by the American Physical Society under the terms of the Creative Commons Attribution 3.0 License. Further distribution of this work must maintain attribution to the author(s) and the published article's title, journal citation, and DOI. functions and is therefore only valid for noninteracting systems. Generalizations involve twisted boundary conditions [21] or Green functions [22-27] and are challenging to use in experiments or exact simulations. Indirect signatures such as the closing of gaps [16] or the crossing of energy levels [28] require, among other difficulties, experimental tuning of microscopic parameters.

Topological insulators show a unique response to topological defects such as dislocations $[29,30]$ or $\pi$ fluxes $[12,30,31]$. Upon adiabatic insertion of a $\pi$ flux, Faraday's law, together with the quantized transverse conductivity, gives rise to midgap charge and spin-fluxon states [12,31]. These states are exponentially localized around the flux $[12,31]$. The existence of these states is ensured, even in the presence of interactions or disorder, by time-reversal symmetry, and has been suggested as a bulk probe of the $Z_{2}$ index [12,31]. The concept of fluxons can also be generalized to situations where spin is not conserved, such as in the presence of Rashba coupling. In three dimensions, a magnetic flux gives rise to the wormhole effect [32]. Electron-electron repulsion lifts the degeneracy of charge and spin fluxons, but the two degenerate spin-fluxon states constitute a localized spin with $S^{z}= \pm 1 / 2$ [12]. Dynamical $\pi$ fluxes emerge in the context of fractionalized topological insulators $[12,13]$.

Previous work on $\pi$ fluxes in noninteracting quantum spin Hall insulators [12,30,31] was based on square-lattice models such as that for HgTe quantum wells [8]. Here, we consider the half-filled Kane-Mele model on the honeycomb lattice [3] (historically the first model with a $Z_{2}$ topological phase), which has close connections to graphene [3], the integer quantum Hall effect [33], and, when including interactions, to correlated Dirac fermions [15]. Topological phases of interacting fermions on honeycomb lattices may be realized in transition metal oxides [34], 
semiconductor structures [35], graphene [36], or cold atoms [37] (see also Ref. [10]).

Here, we use $\pi$ fluxes in combination with exact quantum Monte Carlo simulations and show that they can be used efficiently to probe the topological invariant of correlated topological insulators. In particular, this method does not rely on an adiabatic connection to a noninteracting state, and it may also be used for fractional states. In addition, we demonstrate that $\pi$ fluxes permit the construction of quantum spin models of almost arbitrary geometry and with tunable, dynamical interactions. The spins correspond to the spin fluxons created by inserting $\pi$ fluxes, and the interaction is mediated by magnetic excitons corresponding to collective magnetic fluctuations of the topological insulator. These spin models can be studied theoretically with the quantum Monte Carlo method, or experimentally. As examples, we show that a ring of three spins has a ground state with magnetization $1 / 2$ and that a one-dimensional chain of fluxons undergoes a Mott transition and is described at low energies by an XXZ model.

The article is organized as follows. In Sec. II, we introduce the Kane-Mele and Kane-Mele-Hubbard models. Section III provides details about the methods. The use of $\pi$ fluxes as a probe for topological states is discussed in Sec. IV, whereas the construction of quantum spin models is the topic of Sec. V. Conclusions are given in Sec. VI, and we provide three appendixes.

\section{MODEL}

The half-filled Kane-Mele model with additional electron-electron interactions can be studied with powerful quantum Monte Carlo methods [16,18]. Using the spinor notation $\hat{c}_{i}^{\dagger}=\left(\hat{c}_{i \dagger}^{\dagger}, \hat{c}_{i \downarrow}^{\dagger}\right)$, where $\hat{c}_{i \sigma}^{\dagger}$ is a creation operator for an electron in a Wannier state at site $i$ with spin $\sigma$, the Hamiltonian reads

$$
\begin{aligned}
H_{\mathrm{KM}}= & -t \sum_{\langle i, j\rangle} \tau_{i j} \hat{c}_{i}^{\dagger} \hat{c}_{j}+\mathrm{i} \lambda \sum_{\langle i, j\rangle} \tau_{i j} \hat{c}_{i}^{\dagger}\left(\boldsymbol{\nu}_{i j} \cdot \boldsymbol{\sigma}\right) \hat{c}_{j} \\
& +\mathrm{i} \alpha \sum_{\langle i, j\rangle} \tau_{i j} \hat{c}_{i}^{\dagger}\left(\boldsymbol{s} \times \hat{\boldsymbol{d}}_{i j}\right) \cdot \hat{z} \hat{c}_{j} .
\end{aligned}
$$

The notations $\langle i, j\rangle$ and $\langle i, j\rangle\rangle$ indicate that the sites $i$ and $j$ are nearest neighbors and next-nearest neighbors, respectively, and implicitly include the Hermitian conjugate terms.

The first term describes the hopping of electrons between neighboring lattice sites. The second term represents the spin-orbit coupling which reduces the $S U(2)$ spin rotation symmetry to a $U(1)$ symmetry. The third term is an additional Rashba spin-orbit coupling [38]. The additional factors $\tau_{i j}= \pm 1$ take into account any $\pi$ fluxes present, whereas the original Kane-Mele model (without $\pi$ fluxes) is recovered from Eq. (1) by setting $\tau_{i j}=1$.

The spin-orbit term corresponds to a next-nearestneighbor hopping with a complex amplitude $i \lambda$ and has been derived from the spin-orbit coupling in graphene [3]. This hopping acquires a sign \pm 1 , depending on its direction, the sublattice, and the electron spin. This sign is encoded in $\left(\boldsymbol{\nu}_{i j} \cdot \boldsymbol{\sigma}\right)$, where

$$
\boldsymbol{\nu}_{i j}=\frac{\boldsymbol{d}_{i k} \times \boldsymbol{d}_{k j}}{\left|\boldsymbol{d}_{i k} \times \boldsymbol{d}_{k j}\right|} .
$$

$\boldsymbol{d}_{i k}$ is the vector connecting sites $i$ and $k$, and $k$ is the intermediate lattice site involved in the hopping process from $i$ to $j$. For a coordinate-independent representation, the vectors $\boldsymbol{d}_{\alpha \beta}$ are defined in three-dimensional space, although the $z$ component vanishes. The vector $\boldsymbol{\sigma}$ is defined by $\boldsymbol{\sigma}=\left(\sigma^{x}, \sigma^{y}, \sigma^{z}\right)$, with the Pauli matrices $\sigma^{\alpha}$.

The last term in Eq. (1) is the Rashba spin-orbit interaction [3,5]. It is defined in terms of the spin vector $\boldsymbol{s}=\boldsymbol{\sigma} / 2$ and the unit vector $\hat{\boldsymbol{d}}_{i j}$, which can be expressed in terms of the nearest-neighbor vectors $\boldsymbol{\delta}_{1}, \boldsymbol{\delta}_{2}, \boldsymbol{\delta}_{3}$ [39]. The Rashba coupling breaks the $z \mapsto-z$ inversion symmetry and has to be taken into account, for example, in the presence of a substrate. Because this term includes spin-flip terms, spin is no longer conserved. The Rashba term has been included in the results for the noninteracting model (1), but cannot be included in quantum Monte Carlo simulations of the interacting model (3) due to a minussign problem.

The model (1) can be solved exactly $[3,5,40]$. In the absence of Rashba coupling, $\alpha=0$, the Kane-Mele model describes a $Z_{2}$ quantum spin Hall insulator for any $\lambda>0$. This state is characterized by a bulk band gap $\Delta_{\mathrm{sp}}=3 \sqrt{3} \lambda$, a spin gap $\Delta_{s}=2 \Delta_{\text {sp }}$, and a quantized spin Hall conductivity $\sigma_{x y}^{s}=\frac{e^{2}}{2 \pi}$. The topological state survives for Rashba interactions $\alpha<2 \sqrt{3} \lambda$ (for chemical potential $\mu=0$ ) and has protected, helical edge states for geometries with open boundaries $[3,5,40]$. We use $t$ as the unit of energy $(\hbar=1)$, take $\lambda / t=0.2$, and consider periodic lattices with $L \times L^{\prime}$ unit cells.

To investigate the effect of electron-electron repulsion, we consider the paradigmatic Hubbard interaction [41] and arrive at the Kane-Mele-Hubbard model [42],

$$
H_{\mathrm{KMH}}=H_{\mathrm{KM}}+H_{U}, \quad H_{U}=\frac{1}{2} U \sum_{i}\left(\hat{c}_{i}^{\dagger} \hat{c}_{i}-1\right)^{2} .
$$

Hamiltonian (3) without Rashba coupling has been studied intensely [16,42-48]. In particular, its symmetries permit the application of exact quantum Monte Carlo methods without a sign problem $[16,43,48]$.

On a lattice with periodic boundaries, $\pi$ fluxes can only be inserted in pairs, as illustrated for the minimal number of two fluxes in Fig. 1. The flux pair is connected by a branch cut (or string), and every hopping process crossing the cut acquires a phase $e^{\mathrm{i} \pi}=-1$, as encoded by $\tau_{i j}$ in Eq. (1). Different choices of the branch cut for fixed flux positions are related by a gauge transformation. 


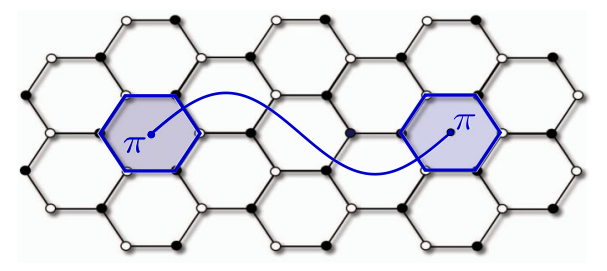

FIG. 1. For a lattice with periodic boundaries, $\pi$ fluxes can be inserted in pairs. Each flux threads a hexagon (highlighted in blue) of the honeycomb lattice, and the pair is connected by a branch cut (blue line). Hopping processes crossing the branch cut acquire a phase $e^{\mathrm{i} \pi}=-1$.

\section{METHOD}

We have used the auxiliary-field quantum Monte Carlo method [49], which was previously applied to the Hubbard model on the honeycomb lattice [15], and the Kane-Mele-Hubbard model [16,43,48]. The central idea of this stochastic method is to use a path integral representation of the interacting model (3). By means of a HubbardStratonovich transformation, the Hubbard term is decoupled, leading to a problem of noninteracting fermions in an external, space-dependent and imaginary-time-dependent field. The sampling is over different configurations of these auxiliary fields in terms of local updates. For a given configuration of fields, Wick's theorem can be used to calculate arbitrary correlation functions from the singleparticle Green function. We refer to a review [50] and previous work $[15,16,48]$ for technical details such as the calculation of energy gaps.

Here, we have used a projective formulation (with projection parameter $\theta t=40$ ) to obtain ground-state results, starting from a trial wave function (the ground state of the $U=0$ case) [48] and a finite-temperature formulation to calculate thermodynamic properties. Both variants rely on a Trotter discretization of imaginary time (we used $\Delta \tau=\beta / L=\theta / L=0.1$ ), but the associated systematic error is smaller than the statistical errors. At half filling, time-reversal invariance ensures that simulations can be carried out without a minus-sign problem, even in the presence of $\pi$ fluxes.

\section{USING $\boldsymbol{\pi}$ FLUXES TO PROBE CORRELATED TOPOLOGICAL STATES}

\section{A. Thermodynamic signature of $\boldsymbol{\pi}$ fluxes}

In the topological phase of the model (1), each $\pi$ flux gives rise to four fluxon states which are exponentially localized (due to the bulk energy gap $\Delta_{\mathrm{sp}}$ ) near the corresponding flux-threaded hexagons [12,31] (see Fig. 1). The states correspond to the spin fluxons $|\uparrow\rangle,|\downarrow\rangle$ (with energy $E^{\Uparrow \downarrow}$ ), forming a Kramers pair related by time reversal, and the charge fluxons $|+\rangle,|-\rangle$ (with energies $E^{+}, E^{-}$), related by particle-hole symmetry. As we show in Fig. 2, the fluxon states lie inside the bulk band gap, and for noninteracting electrons, $E^{\Uparrow \downarrow}=E^{+}=E^{-}$.
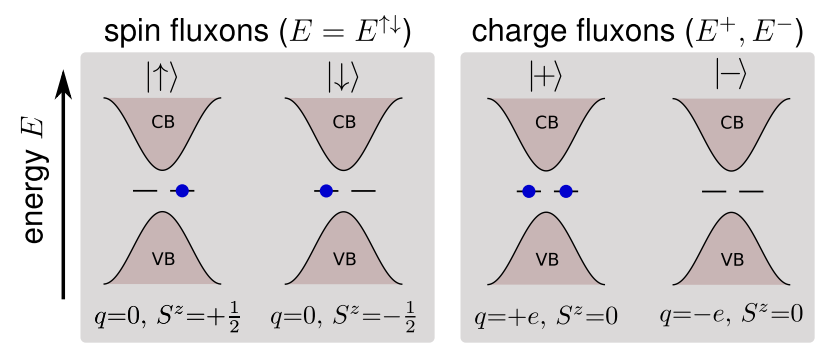

FIG. 2. In a quantum spin Hall insulator, a $\pi$ flux gives rise to four states (with charge $q$ and spin $S^{z}$ ) localized near the flux, which lie inside the bulk energy gap between the valence and conduction bands (labeled "VB" and "CB" in the figure, respectively) [12,31]. The states correspond to a Kramers doublet of spin fluxons $|\uparrow\rangle,|\downarrow\rangle$ with energy $E^{\uparrow \downarrow}$ and a doublet of charge fluxons $|+\rangle,|-\rangle$ with energies $E^{+}, E^{-}$.

The fluxons leave a characteristic signature in the static spin and charge susceptibilities,

$$
\chi_{s}=\beta\left(\left\langle\hat{M}_{z}^{2}\right\rangle-\left\langle\hat{M}_{z}\right\rangle^{2}\right), \quad \chi_{c}=\beta\left(\left\langle\hat{N}^{2}\right\rangle-\langle\hat{N}\rangle^{2}\right),
$$

which are defined in terms of the operators of total spin in the $z$ direction $\left(\hat{M}_{z}=\sum_{i} \hat{c}_{i}^{\dagger} \sigma^{z} \hat{c}_{i}\right)$ and of the total charge $\left(\hat{N}=\sum_{i} \hat{c}_{i}^{\dagger} \hat{c}_{i}\right)$; the inverse temperature is given by $\beta=\frac{1}{k_{B} T}$. At low temperatures, $k_{B} T \ll \Delta_{\mathrm{sp}}$, we can restrict the Hilbert space to $\{|\uparrow\rangle,|\downarrow\rangle,|+\rangle,|-\rangle\}$. If the spin fluxons are independent, and for $\alpha=0$, we expect a Curie law $\chi_{s}=\chi_{c}=\frac{1}{2 k_{B} T}$ per $\pi$ flux, and hence $\chi_{s}=\chi_{c}=\frac{1}{k_{B} T}$ for two independent $\pi$ fluxes (see Appendix A). The prefactor of the Curie law follows from the quantized spin Hall conductance in the absence of Rashba coupling [3]. Similarly, a Curie law was also predicted for topological excitations in polyacetylene [51].

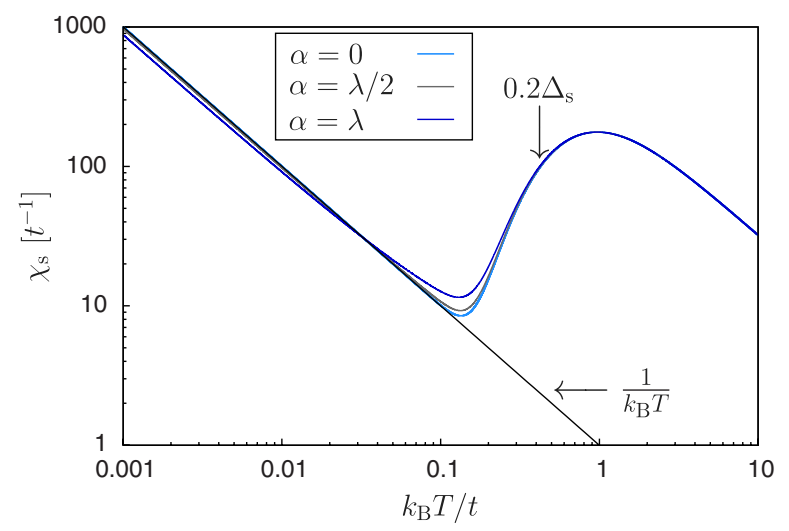

FIG. 3. Spin susceptibility of the Kane-Mele model $(\lambda / t=0.2)$ with two $\pi$ fluxes at the maximal distance on an $18 \times 18$ lattice, for different Rashba couplings $\alpha$. At temperatures $k_{B} T \lesssim 0.1 t$, each $\pi$ flux contributes $\frac{1}{2 k_{B} T}$ to the susceptibility, leading to $\chi_{s} \approx \frac{1}{k_{B} T}$. Also shown is the spin-gap energy scale $0.2 \Delta_{s}$ for $T=0, \alpha=0$. For $\alpha>0$, the chemical potential is adjusted to retain a half-filled band. 
Figure 3 shows results for $\chi_{s}$ as a function of temperature for the Kane-Mele model with two $\pi$ fluxes located at the largest possible distance. At temperatures $k_{B} T \approx \Delta_{s}$, $\chi_{s}$ is dominated by bulk effects. For $k_{B} T \lesssim 0.1 t$, we observe the expected Curie law. The latter is robust with respect to Rashba coupling, which is crucial for possible experimental realizations.

\section{B. Probing correlated topological insulators}

Figure 3 establishes the existence and thermodynamic signature of degenerate spin and charge fluxons in a quantum spin Hall insulator threaded by a pair of $\pi$ fluxes. We now consider the effect of electron-electron interactions in the framework of the Kane-Mele-Hubbard model (3). For $\lambda>0$, the phase diagram of the latter includes a correlated quantum spin Hall insulating phase that is adiabatically connected to that of the Kane-Mele model (i.e., $U=0$ ), and a Mott insulating phase with long-range antiferromagnetic order $[42,48]$. Figure 4(a) shows the quantum phase transition between these two phases as a function of $U / t$ at $\lambda / t=0.2$. At the transition, the spin gap $\Delta_{s}$-as obtained from finite-size scaling (see Ref. [48] for details)—closes, corresponding to the condensation of magnetic excitons $[47,48]$. The magnetic order is of the easy-plane type, and the transition has 3D XY universality corresponding to the ordering of local moments [47,48]. For $U \geq U_{c}$,
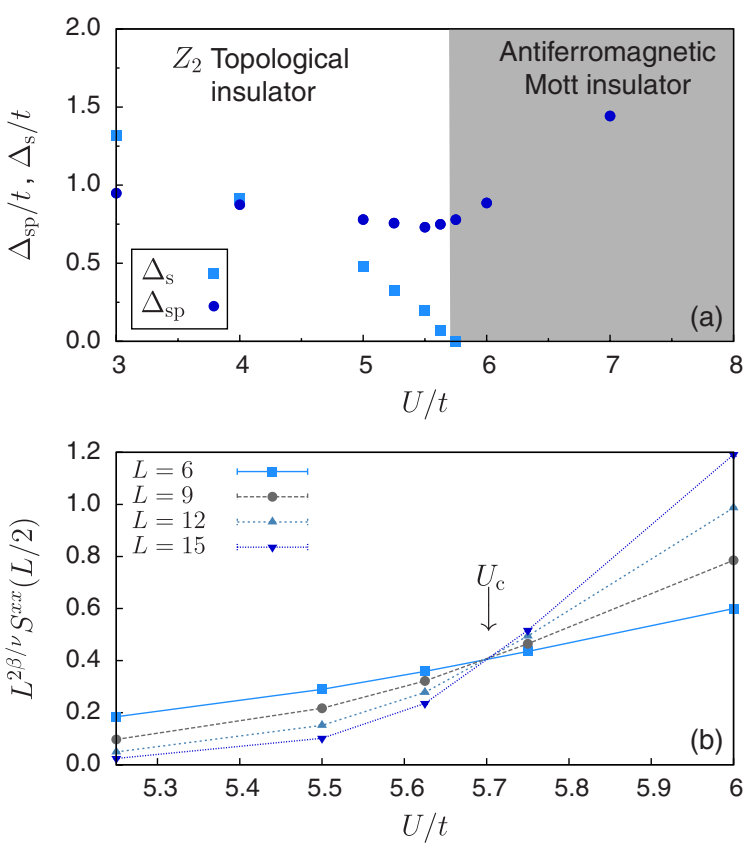

FIG. 4. (a) Spin gap $\Delta_{s}(\boldsymbol{q}=0)$ and single-particle gap $\Delta_{\mathrm{sp}}(\boldsymbol{q}=\boldsymbol{K})$ in the thermodynamic limit as a function of the Hubbard repulsion $U$, at $T=0(\lambda / t=0.2, \alpha=0)$. (b) Scaling of $S^{x x}(L / 2)$ using the critical exponents of the 3D XY model, $z=1, \nu=0.6717(1)$, and $\beta=0.3486(1)$ [52]. The intersection gives the critical point $U_{c} / t=5.70(3)$. The lattice size is $L \times L$. Error bars are smaller than the symbols. time-reversal symmetry is spontaneously broken, and the single-particle gap $\Delta_{\mathrm{sp}}$ remains open across the transition [48] [see Fig. 4(a)].

The location of the critical point can be estimated from the scaling behavior of the real-space spin-spin correlation function

$$
S^{x x}(r)=\left\langle S_{A}^{x}(r) S_{A}^{x}(0)\right\rangle
$$

at the largest distance $r=L / 2$. Here, we consider correlations between spins on the $A$ sublattice, but results are the

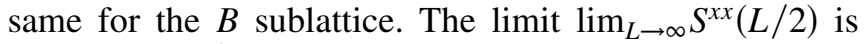
identical to $m^{2}$, with $m$ being the magnetization per site. This critical value can be obtained by considering the 3D XY scaling behavior at the transition. Following Ref. [48], we plot $L^{2 \beta / \nu} S^{x x}(L / 2)$ as a function of $U$ for different system sizes by using the critical exponents $z=1, \nu=$ $0.6717(1)$, and $\beta=0.3486(1)$ [52]. Figure 4(b) reveals the expected intersection of curves at the critical point and gives $U_{c} / t=5.70(3)$.

The well-understood magnetic transition of the model (3) provides a test case for the use of $\pi$ fluxes to probe a correlated quantum spin Hall state, as well as to track the interaction-driven transition to a topologically trivial state. We solve the interacting model with two $\pi$ fluxes by using exact quantum Monte Carlo simulations. Spin fluxons can be detected by calculating the lattice-site-resolved, dynamical spin-structure factor at $T=0$, defined as

$$
S(i, \omega)=\pi \sum_{n}\left|\left\langle n\left|\hat{c}_{i}^{\dagger} \sigma^{z} \hat{c}_{i}\right| 0\right\rangle\right|^{2} \delta\left(E_{n}-E_{0}-\omega\right) .
$$

Here, $H_{\mathrm{KMH}}|n\rangle=E_{n}|n\rangle$, and $|0\rangle$ denotes the ground state. $S(i, \omega)$ corresponds to the spectrum of spin excitations at lattice site $i$. A real-space map of the spin-fluxon states $|\uparrow\rangle$, $|\downarrow\rangle$ is obtained by integrating $S(i, \omega)$ up to an energy scale $\Omega / t=0.2$, well within the charge gap $\Delta_{c} \approx 2 \Delta_{\mathrm{sp}}$, giving $S_{\Omega}(i)=\int_{0}^{\Omega} \mathrm{d} \omega S(i, \omega)$. For $U / t=4$, corresponding to the quantum spin Hall phase [see Fig. 4(a)], we see in Fig. 5(a) very sharply defined spin fluxons localized at the two fluxthreaded hexagons. The value of $S_{\Omega}(i)$ is about 3 orders of magnitude smaller at lattice sites that are further away from a flux so that the spin fluxons can easily be detected numerically. In Fig. 5(b), we show results for the magnetic insulating phase at $U / t=6$. As expected for this topologically trivial state, no well-defined spin fluxons exist.

The dependence of $S_{\Omega}(i)$ on $U / t$ across the magnetic quantum phase transition is shown in Fig. 5(c). A clear signal is found deep in the topological-insulator phase, whereas a strong drop is observed on approaching the critical point at $U_{c} / t=5.70(3)$. Hence, the spin-fluxon signal can be used in quantum Monte Carlo simulations to distinguish topological and nontopological phases.

As for the noninteracting case (Fig. 3), the spin fluxons created by the fluxes give rise to a characteristic Curie law in the spin susceptibility. Figure 6(a) shows quantum Monte Carlo results for the spin and charge susceptibilities 
(a) Topological insulator $(U / t=4)$

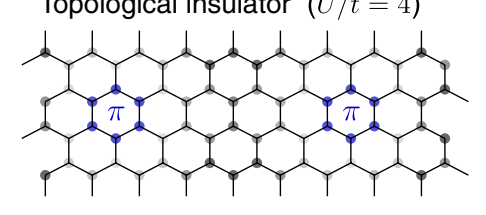

$S_{\Omega}(i)\left[t^{-1}\right]$

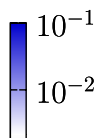

(b)
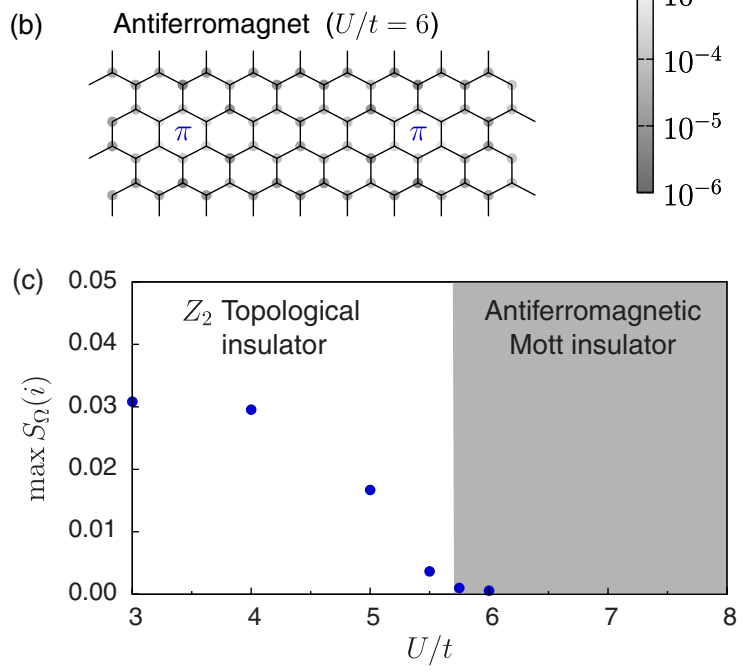

FIG. 5. Integrated dynamical spin-structure factor $S_{\Omega}(i)$ at $T=0$ on a $9 \times 9$ lattice. (a) Localized spin fluxons created in the topological-insulator phase at $U / t=4$. (b) Absence of spin fluxons in the magnetic phase at $U / t=6$. (c) Maximum of $S_{\Omega}(i)$, as a function of $U / t$. Here $\lambda / t=0.2, \alpha=0$, and $\Omega / t=0.2$.

defined in Eq. (4) in the topological phase $(U / t=4)$. We again consider two fluxes at the maximal separation. At low temperatures, $k_{B} T \ll \Delta_{s}, \chi_{s}$ is well described by $\chi_{s}=\frac{2}{k_{B} T}$, or $\frac{1}{k_{B} T}$ per $\pi$ flux. The additional factor of 2 compared to the case $U=0$ comes from the splitting of spin and charge states, which only leaves the Kramers doublet $\{|\uparrow\rangle,|\downarrow\rangle\}$ at low energies (see Appendix A). The Curie law holds down to the lowest temperatures considered in Fig. 6(a). Finally, the charge susceptibility is strongly suppressed at low temperatures and reveals the absence of low-energy charge fluxons as a result of the Hubbard repulsion.

\section{Interaction between spin fluxons}

So far, we have exploited the thermodynamic and spectral signatures of independent spin-fluxon excitations (i.e., free spins). On periodic lattices, spin fluxons can only be created in pairs, and it is therefore interesting to consider their mutual interactions. Such interactions will play a key role in Sec. V, where we consider quantum spin Hall insulators with multiple $\pi$ fluxes to create and simulate systems of interacting spins.

Interaction effects due to a coupling between two spin fluxons in a lattice with one pair of $\pi$ fluxes become visible for larger $U / t$, i.e., closer to the magnetic transition. Figures 6(b) and 6(c) show a deviation from the Curie law below a temperature scale determined by the interaction between spin fluxons. In the model (3), this interaction is mediated by the exchange of collective spin excitations (magnetic excitons), which are the lowest-lying excitations of the correlated topological insulator, and they evolve into the gapless Goldstone mode of the magnetic state. Since magnetic order is of the easy-plane type, the dominant contribution of the resulting interaction is expected to have the general form

$$
\begin{aligned}
S_{\text {int }}= & -g^{2} \sum_{\boldsymbol{r} \neq \boldsymbol{r}^{\prime}} \iint_{0}^{\beta} \mathrm{d} \tau \mathrm{d} \tau^{\prime}\left[\mathcal{S}_{\boldsymbol{r}}^{+}(\tau) D\left(\boldsymbol{r}-\boldsymbol{r}^{\prime}, \boldsymbol{\tau}-\tau^{\prime}\right)\right. \\
& \left.\times \mathcal{S}_{\boldsymbol{r}^{\prime}}^{-}\left(\tau^{\prime}\right)+\text { H.c. }\right],
\end{aligned}
$$

where $\mathcal{S}_{r}^{ \pm}(\tau)$ are spin-flip operators acting on a spin fluxon at position $\boldsymbol{r}$ at time $\tau, D\left(\boldsymbol{r}-\boldsymbol{r}^{\prime}, \tau-\tau^{\prime}\right)$ is the Fourier
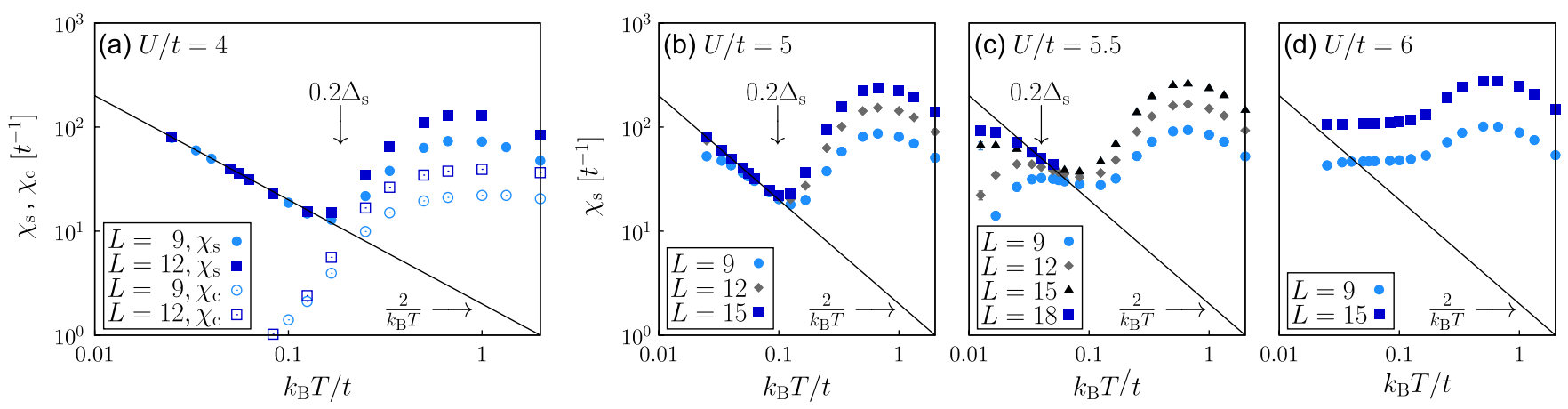

FIG. 6. (a) Spin $\left(\chi_{s}\right)$ and charge $\left(\chi_{c}\right)$ susceptibilities of the Kane-Mele-Hubbard model $(\lambda / t=0.2, \alpha=0)$ at $U / t=4$. We consider $L \times L$ lattices with one pair of $\pi$ fluxes placed at the maximal distance. At low temperatures, the spin susceptibility reveals a Curie law $\chi_{s}=\frac{2}{k_{B} T}$, whereas the charge susceptibility is suppressed by the charge gap. (b), (c) Spin susceptibility as a function of temperature for different values of $U / t(\lambda / t=0.2, \alpha=0)$. (a)-(c) show that with increasing $U / t$, the range of the interaction between spin fluxons increases, leading to deviations from the Curie law $\chi_{s}=\frac{2}{k_{B} T}$ at low temperatures. (d) For $U>U_{c}=5.70(3) t, \chi_{s}$ reflects the presence of long-range magnetic order in the bulk. Error bars are smaller than the symbol size. The arrows indicate the energy scale associated with the spin gap. 
transform of the exciton propagator $D\left(\boldsymbol{q}, \mathrm{i} \Omega_{m}\right)(\boldsymbol{q}$ : momentum; $\Omega_{m}=2 n \pi / \beta$ : bosonic Matsubara frequency), and $g$ is a coupling constant. At long wavelengths, the dispersion relation of the collective spin mode can be written as $\omega(\boldsymbol{q})=\sqrt{v^{2}|\boldsymbol{q}-\boldsymbol{Q}|^{2}+\Delta_{s}^{2}}$, where $v$ is the spin velocity, $\Delta_{s}$ is the spin gap, and $\boldsymbol{Q}$ is the magnetic-ordering wave vector. The minimal exciton energy is given by $\omega(\boldsymbol{q}=\boldsymbol{Q})=\Delta_{s}$. Fourier transformation of the propagator (see Appendix C) gives, in the limit of low energies and long wavelengths,

$$
D(\boldsymbol{r}, \tau) \sim \exp (\mathrm{i} \boldsymbol{Q} \cdot \boldsymbol{r}) \exp \left(-\Delta_{s} \tau\right) \exp \left(-\frac{|\boldsymbol{r}|^{2} \Delta_{s}}{2 \boldsymbol{v}^{2} \tau}\right)
$$

The first term determines the sign of the interaction. The decay at large imaginary time $\tau$ is governed by the spin gap $\Delta_{s}$. The fast decay as a function of $|\boldsymbol{r}|$ underlies the clear Curie law seen, e.g., in Fig. 6. The interaction range and strength can be tuned via the spin gap and hence [cf. Fig. 4(a)] by varying $U / t$.

From Eq. (8), we expect the interaction range to increase with increasing $U / t$ due to the decrease of $\Delta_{s}$ [see Fig. 4(a)]. Indeed, Figs. 6(b) and 6(c) reveal an enhanced effect of the spin-fluxon separation at low temperatures with increasing $U / t$. In particular, for $U / t=5.5$ (close to the magnetic transition), Fig. 6(c) shows a Curie law corresponding to two free spin fluxons, only for the largest system sizes $(L=18)$. As $U \rightarrow U_{c}$, the interaction range diverges, and free spin fluxons can no longer exist. For $U>U_{c}$, time-reversal invariance is broken, and $\pi$ fluxes do not create spin fluxons. Instead, the spin susceptibility in Fig. 6(d) is that of an antiferromagnet; the finite value of $\chi_{s} / L^{2}$ at $T=0$ reflects the density of spin-wave excitations.

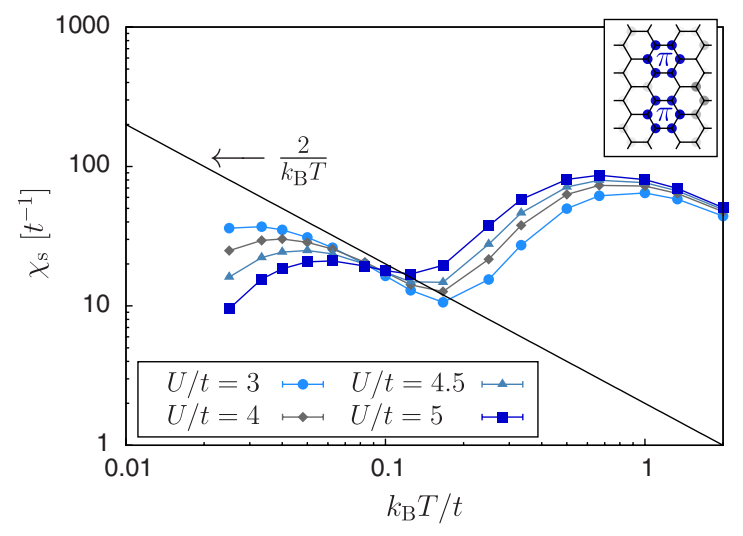

FIG. 7. Spin susceptibility as a function of temperature for two $\pi$ fluxes arranged as shown in the inset $(\lambda / t=0.2, \alpha=0,9 \times 9$ lattice). With increasing $U / t$, the strength of the interaction between spin fluxons increases, as revealed by the shift of the temperature below which deviations from a $\frac{2}{k_{B} T}$ Curie law occur. Statistical errors are smaller than the symbol size. Inset: $S_{\Omega}(i)$ for $U / t=4$, using the same color coding as in Fig. 5(a).
To illustrate the dependence of the interaction strength on $\Delta_{s}$, we consider two fluxes at a fixed, small distance, as illustrated in the inset of Fig. 7. We show the spin susceptibility for different values of $U / t$ in Fig. 7. For $U / t=3$, a Curie law $\chi_{s} \approx \frac{2}{k_{B} T}$ may be inferred at temperatures $k_{B} T \approx$ $0.1 t$. Increasing $U / t$, the interaction between the spin fluxons becomes too large to observe free spin fluxons below the temperature range set by the bulk spin gap $\Delta_{s}$. The downturn of $\chi_{s}$ occurs at higher and higher temperatures with increasing $U / t$, and it reflects a tunable, correlation-induced energy scale for the interaction between spin fluxons that is absent in Fig. 3.

\section{V. $\pi$-FLUX QUANTUM SPIN MODELS}

The possibility of inserting $\pi$ fluxes to create localized spin fluxons with a tunable interaction mediated by magnetic excitons provides a toolbox to engineer interacting spin models in correlated topological insulators. The computational effort for quantum Monte Carlo simulations does not depend on the number of $\pi$ fluxes, and the latter can be arranged in almost arbitrary geometries on the honeycomb lattice.

\section{A. Three-spin system}

As a first extension of the two-spin cases considered so far, we consider four spin fluxons emerging from two pairs of $\pi$ fluxes. The fluxes are arranged so that three spin fluxons form a ring, and the fourth spin fluxon is located at the largest distance from the center of the ring. For large enough lattices, the separated spin fluxon will not couple to the other three, and the physical problem is similar to experiments on coupled quantum dots [53] or flux qubits [54] in the context of quantum computation. The three spin fluxons experience a transverse interaction of the form (7)

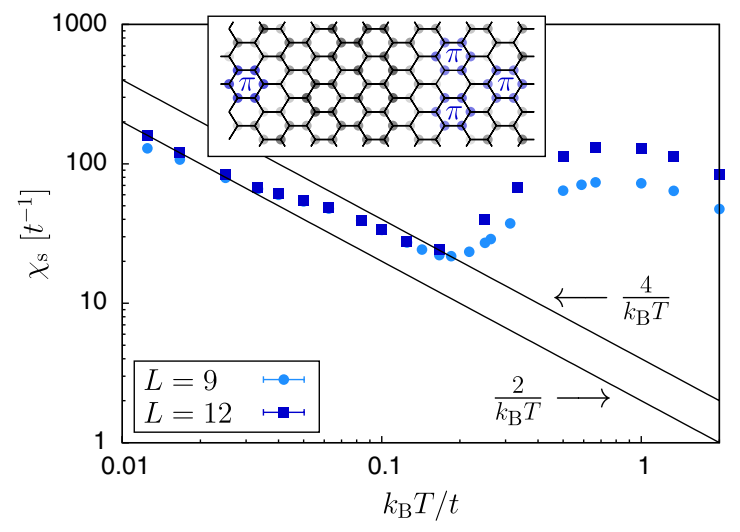

FIG. 8. Spin susceptibility as a function of temperature for four $\pi$ fluxes arranged as shown in the inset $(\lambda / t=0.2, \alpha=0$, $U / t=4)$ on $L \times L$ lattices. The data reveal a Curie law $\frac{4}{k_{B} T}$ at intermediate temperatures and $\frac{2}{k_{B} T}$ at low temperatures. Statistical errors are smaller than the symbol size. Inset: $S_{\Omega}(i)$ for $L=15$, using the same color coding as in Fig. 5(a). 
and behave as an effective spin with $S^{z}= \pm 1 / 2$ at low temperatures (see Appendix B). The spin susceptibility for $U / t=4$ shown in Fig. 8 reveals that, at low temperatures, the two independent spins indeed give rise to the expected Curie law $\chi_{s}=\frac{2}{k_{B} T}$. At higher temperatures $k_{B} T \approx 0.1 t$, we find $\chi_{s}=\frac{4}{k_{B} T}$, corresponding to four independent spin fluxons. In the regime where $\chi_{s}=\frac{2}{k_{B} T}$, the sign of the interaction between the spin fluxons determines the ground-state degeneracy of the three-spin cluster. A net ferromagnetic interaction results in a spin- $1 / 2$ doublet, whereas an antiferromagnetic coupling gives rise to a fourfold degenerate, chiral ground state (see Appendix B). In principle, the sign of the exchange coupling can be determined from entropy measurements. Since $\boldsymbol{Q}=0$ for the model (3), Eq. (8) suggests that the interaction is ferromagnetic.

\section{B. Simulation of one-dimensional fluxon chains}

Whereas the study of systems with a small number of spins is relevant for applications such as quantum computing, many questions in condensed matter physics are related to periodic spin lattices. In this section, we therefore consider one-dimensional chains of $\pi$ fluxes in the honeycomb lattice with periodic boundary conditions.

We begin with the noninteracting Kane-Mele model with a periodic flux chain. The fluxon excitations are visible in Fig. 9, which shows the integrated local density of states, $A_{\Omega}(i)=\int_{0}^{\Omega} d \omega A(i, \omega)$; the single-particle spectral function $A(i, \omega)$ is defined as usual in terms of the single-particle Green function, $A(i, \omega)=-\operatorname{Im} G(i, \omega)$. Whereas the fluxons are well localized in the direction normal to the chain, the overlap of neighboring fluxons in the chain gives rise to a tight-binding band inside the topological band gap, which can be seen in the spectrum shown in Fig. 10. The specific form of the band structure can be attributed to the fact that the smallest unit cell for the fluxon chain contains two flux-threaded hexagons

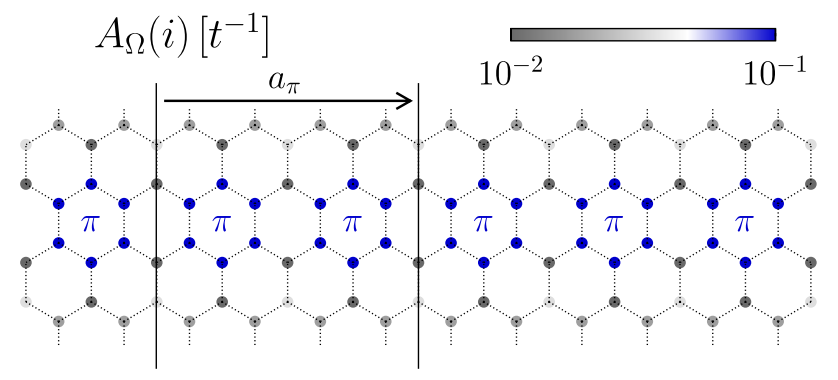

FIG. 9. Integrated local density of states $A_{\Omega}(i)(\Omega=0.2 t$; see text) at $T=0$ for the Kane-Mele model $(\lambda / t=0.2, \alpha=0)$ with a periodic chain of $\pi$ fluxes. We show a part of the $72 \times 12$ lattice used and the size of the magnetic unit cell containing two $\pi$ fluxes. The latter has width $a_{\pi}=4 a$, where $a \equiv 1$ corresponds to the norm of the lattice vectors of the underlying honeycomb lattice.

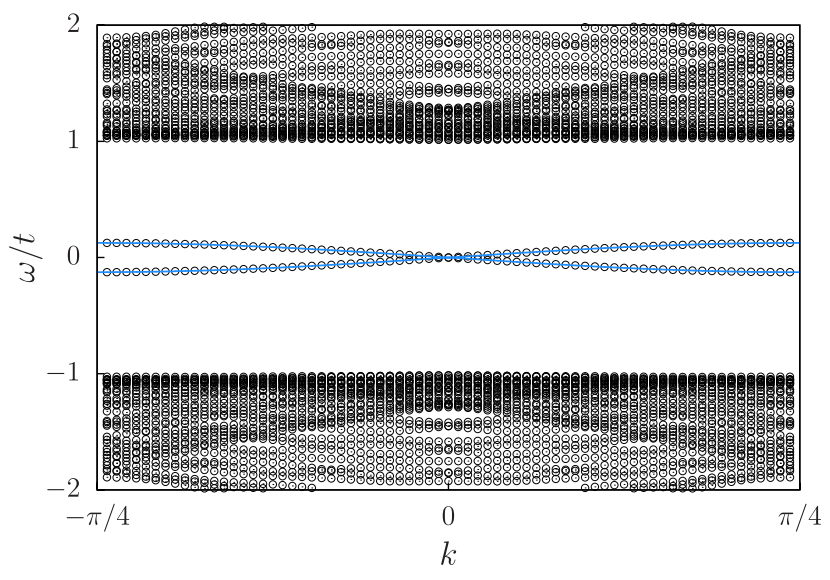

FIG. 10. Spectrum of eigenvalues of the Kane-Mele model with a periodic chain of $\pi$ fluxes (cf. Fig. 9). Here, $\lambda / t=0.2$, $\alpha=0$, and the honeycomb lattice has dimensions $72 \times 12$. Points correspond to eigenvalues and lines to the band structure $\epsilon(k)= \pm 2 \tilde{t} \sin (2 k a)$, with $\tilde{t} \approx 0.126 t$ and $a \equiv 1$.

(and is four hexagons wide) (see Fig. 9). Exploiting the fact that the four possible fluxon states per hexagon, $\{|\uparrow\rangle,|\downarrow\rangle,|+\rangle,|-\rangle\}$, can formally be written in terms of the fermion Fock states $\{|\uparrow\rangle,|\downarrow\rangle,|0\rangle,|\uparrow \downarrow\rangle\}$, and assuming nearest-neighbor hopping, a suitable Hamiltonian is given by

$$
H=-\tilde{t} \sum_{i \sigma}\left(\phi_{i \sigma}^{\dagger} \psi_{i \sigma}-\psi_{i \sigma}^{\dagger} \phi_{i+a_{\pi} \sigma}+\text { H.c. }\right),
$$

where $\phi, \psi$ refer to the two flux-threaded hexagons in the unit cell, and $i$ numbers the unit cells. The resulting band dispersion $\epsilon(k)= \pm 2 \tilde{t} \sin (2 k a)$ matches the lowenergy bands in the spectrum (Fig. 10). The form of the effective low-energy Hamiltonian, and especially the gapless nature of the spectrum, stems from the fact that the unit cell is a gauge choice; a translation by half a lattice vector, $a_{\pi} / 2$, corresponds to a gauge transformation. This symmetry allows the intercell and intracell hopping integrals to differ only by a phase $e^{i \theta}$. Imposing time-reversal symmetry pins the phase factor to $\theta=0$ and $\theta=\pi$, thus leading to the dispersion relations $\pm 2 \tilde{t} \cos \left[\left(k+\theta / a_{\pi}\right) a_{\pi} / 2\right]$. The choice $\theta=\pi$ produces the above-mentioned dispersion relation, and the choice $\theta=0$ merely corresponds to translating the reciprocal lattice by half a reciprocal lattice unit vector.

In contrast to the helical edge states of a quantum spin Hall insulator, each of the two fluxon bands is spin degenerate. As a result, and because the system is half filled, we expect a Mott transition of charge fluxons for any nonzero electron-electron repulsion. Figure 11 shows the spin and charge susceptibilities of the Kane-Mele-Hubbard model on $L \times 12$ lattices with $L / 2 \pi$ fluxes and $U / t=4$. The Hubbard $U$ causes an exponential suppression of the charge susceptibility at low temperatures [see Fig. 11(b) and inset], whereas low-energy spin-fluxon excitations 

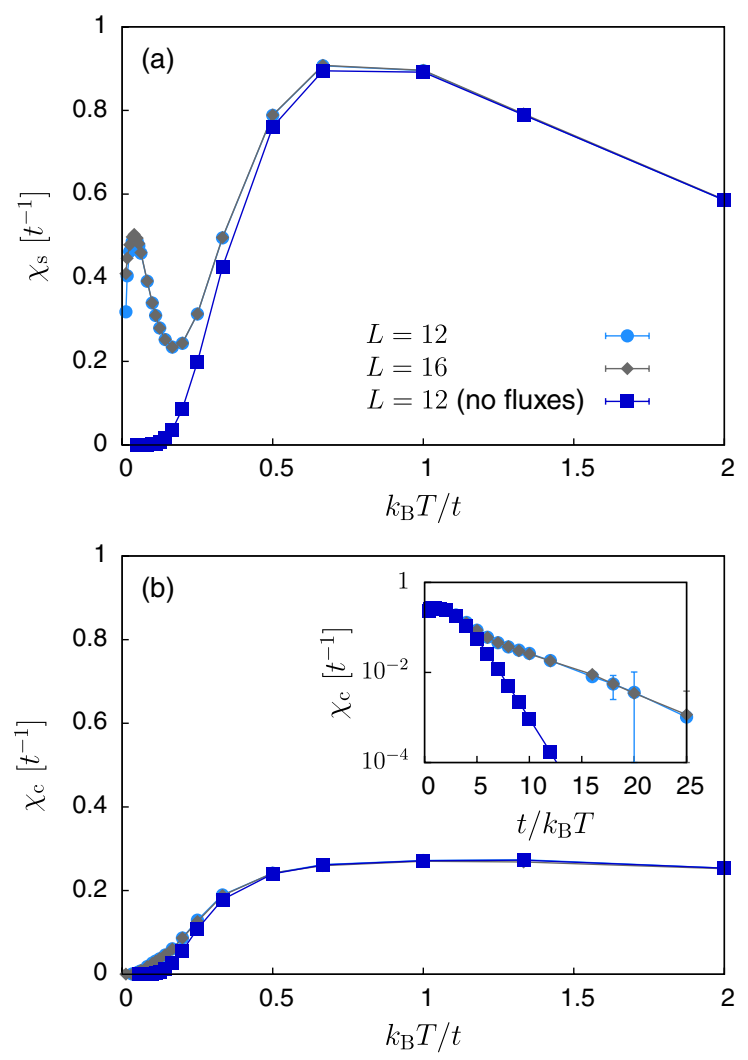

FIG. 11. (a) Spin and (b) charge susceptibility of the Kane-Mele-Hubbard model $(\lambda / t=0.2, \alpha=0)$ at $U / t=4$. We consider $L \times 12$ lattices with $L / 2 \pi$ fluxes arranged in a periodic one-dimensional chain. The inset in (b) shows the charge susceptibility as a function of inverse temperature on a logarithmic scale. The key in (a) applies to all panels.

remain [Fig. 11(a)]. Hence, similar to the one-dimensional Hubbard model, the fluxon chain undergoes a Mott transition to a state with a nonzero charge gap but gapless spin excitations.

In the Mott phase of the fluxon chain, the low-energy physics is expected to be described by spin fluctuations and hence by an effective spin model with spins corresponding to Kramers doublets of localized spin fluxons. Because the interaction range depends exponentially on the spin gap, we expect nearest-neighbor interactions $J^{x y}, J^{z z}$ between spin fluxons to dominate, except for the close vicinity of the magnetic transition. As argued before, the magnetic exciton is of predominantly easy-plane type, and we therefore expect anisotropic interactions, $\left|J^{x y}\right| \gg\left|J^{z z}\right|$. The minimal model for the spin chain is the one-dimensional XXZ Hamiltonian,

$$
H=J^{z z} \sum_{i} S_{i}^{z} S_{i+1}^{z}+J^{x y} \sum_{i}\left(S_{i}^{+} S_{i+1}^{-}+S_{i}^{-} S_{i+1}^{+}\right) .
$$

Using the ALPS 1.3 implementation [55], we can simulate this model in the stochastic-series-expansion representation to calculate the spin susceptibility as a function of temperature. There is one free parameter, $J^{x y} / J^{z z}$, which

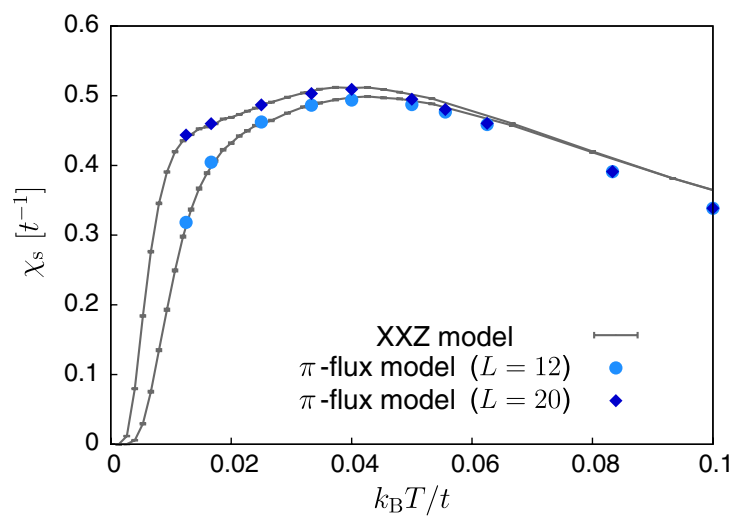

FIG. 12. Spin susceptibility as a function of temperature. Symbols correspond to quantum Monte Carlo results for the Kane-Mele-Hubbard model $(\lambda / t=0.2, \alpha=0, U / t=4)$ on $L \times 12$ lattices with $L / 2 \pi$ fluxes arranged in a periodic onedimensional chain. Lines are quantum Monte Carlo results for the one-dimensional XXZ model with $J^{z z} /\left|J^{x y}\right|=-0.1$ and $L / 2$ lattice sites (spins).

is varied to obtain the best fit to the low-temperature susceptibility (at high temperatures, bulk states of the topological insulator begin to play a role) of the KaneMele-Hubbard model. For example, considering six spins, a rather good match between the spin-fluxon data and the XXZ model is obtained for $J^{z z} /\left|J^{x y}\right|=-0.1$ (the sign of $J^{x y}$ is irrelevant) (see Fig. 12). Importantly, taking the same parameters, and simulating ten spins with both spin fluxons and the XXZ model, equally good agreement is found in Fig. 12. These results demonstrate that the spin fluxons form a one-dimensional spin system with well-defined interactions and that a quantum spin Hall insulator with $\pi$ fluxes can indeed be used as a quantum simulator.

\section{CONCLUSIONS}

In this work, we have presented quantum Monte Carlo results for a correlated quantum spin Hall insulator with topological defects in the form of $\pi$ fluxes. Such fluxes represent a universal probe for the topological index that can be used in the presence of electronic correlations and does not rely on spin conservation or an adiabatic connection to a noninteracting topological insulator. Our results demonstrate that $\pi$ fluxes can be combined with exact numerical simulations and lead to clear signatures of nontrivial topological properties in spectral and thermodynamic properties. As a concrete example, we have studied the magnetic quantum phase transition of the Kane-MeleHubbard model at which time-reversal symmetry is spontaneously broken. In principle, $\pi$ fluxes can also be used in connection with fractional quantum spin Hall states.

More generally, $\pi$ fluxes in correlated topological insulators allow one to construct and simulate quantum spin models and hence lead to a novel class of quantum 
simulators. This finding is not restricted to the Kane-MeleHubbard model considered here. In particular, magnetism driven by electronic correlations - the origin of the interaction between spin fluxons-is a common phenomenon. The physics described here relies on the coexistence of magnetic correlations and time-reversal symmetry and cannot be captured by static mean-field descriptions. The spin models share the topological protection of their host against, for example, disorder. In general, they are characterized by a dynamical, time-dependent interaction reminiscent of spin-boson problems. The detailed form and sign of the interaction, whose strength and range can be tuned via the electronic interactions, depend on the electronic Hamiltonian and the lattice geometry of the underlying topological insulator. Because of the spin-orbit interaction, the spin symmetry is $U(1)$, and-similar to cold-atom realizations of the quantum Ising model [56]- the spinspin interaction is generically anisotropic. We have provided explicit evidence for the feasibility of our idea in terms of simulations of two and four spins, as well as of one-dimensional spin chains. Additional Rashba terms lead to spin models with a discrete $Z_{2}$ Ising symmetry. Although spin-fluxon states are still well defined [12,31], it is a priori not clear which operators have to be measured in the numerical simulations. Finally, the concept of fluxons originating from $\pi$ fluxes carries over to three-dimensional topological insulators [12,32].

An open question of central importance is whether the use of $\pi$ fluxes will enable us to study quantum spin systems that are currently not accessible to numerical methods, for example, due to a sign problem in the presence of frustrated interactions. Whereas we have provided evidence for the possibility to simulate arrays and chains of quantum spins, and to tune the interaction strength and range, entropy measurements are required to determine the sign of the interactions. However, the latter are extremely demanding to carry out using discrete-time quantum Monte Carlo methods. A systematic effort to study spin fluxon chain and ladder geometries is currently in progress.

Our idea may potentially also be used in experiments. A strongly correlated topological insulator on the honeycomb lattice may be realized with $\mathrm{Na}_{2} \mathrm{IrO}_{3}$ [34] or with molecular graphene [57]. It has been suggested that $\pi$ fluxes can be created in a quantum spin Hall insulator by means of an adjacent superconductor and a magnetic field [31]. This idea can be generalized to arrays of $\pi$ fluxes using Abrikosov lattices. Alternatively, $\pi$ fluxes may be realized using SQUIDs. A potential problem is that the diameter of the $\pi$ fluxes will not be of the order of the lattice constant. Other exciting recent proposals that are relevant for the realization of our idea include artificial semiconductor honeycomb structures [35], cold atoms in optical lattices [19], and cold atoms on chips [58]. In solidstate setups, $\pi$ fluxes can also be created by dislocations $[29,30]$ or wedge disclinations [59].

\section{ACKNOWLEDGMENTS}

We thank N. Cooper, T. L. Hughes, L. Molenkamp, J. Moore, J. Oostinga, X.-L. Qi, C. Xu, and S.C. Zhang for insightful conversations, and acknowledge support from DFG Grants No. FOR1162 and No. As 120/4-3, as well as generous computer time at the LRZ Munich and the JSC. We made use of ALPS 1.3 [55].

\section{APPENDIX A: SPIN SUSCEPTIBILITY FOR TWO $\pi$ FLUXES}

A single $\pi$ flux in a topological insulator gives rise to four states, $|\uparrow\rangle,|\downarrow\rangle,|+\rangle,|-\rangle$. In the absence of correlations, these states are degenerate. At low temperatures, the spin susceptibility, defined in Eq. (4), can be calculated using the Hilbert space formed by only these states. Defining an effective Hamiltonian $H_{\pi}=\sum_{\psi} E^{\psi}|\psi\rangle\langle\psi|$ with $\psi \in\{+,-, \uparrow, \downarrow\}$ and $E^{+}=E^{-}=E^{\uparrow}=E^{\downarrow}=E^{\Uparrow \downarrow}$, we obtain

$$
\chi_{s}=\beta\left(\left\langle\hat{M}_{z}^{2}\right\rangle-\left\langle\hat{M}_{z}\right\rangle^{2}\right)=\frac{1}{k_{B} T} \frac{\sum_{\psi}\left\langle\psi\left|\hat{M}_{z}^{2} e^{-\beta H_{\pi}}\right| \psi\right\rangle}{\sum_{\psi}\left\langle\psi\left|e^{-\beta H_{\pi}}\right| \psi\right\rangle}=\frac{1}{2 k_{B} T} .
$$

For $U \gg k_{B} T$, the spin fluxons $|\uparrow\rangle,|\downarrow\rangle$ are the only lowenergy excitations, and $\chi_{s}$ can be calculated by restricting $\psi$ to $\{\uparrow, \downarrow\}$. Since $E^{\uparrow}=E^{\downarrow}=E^{\uparrow \downarrow}$ due to time-reversal symmetry, we get

$$
\chi_{s}=\frac{1}{k_{B} T} .
$$

For the case of two independent $\pi$ fluxes, the above results imply $\chi_{s}=\frac{1}{k_{B} T}$ at $U=0$ and $\chi_{s}=\frac{2}{k_{B} T}$ for $U>0$. These results agree with the numerical results shown in Fig. 3 for $U=0$ and in Figs. 6 and 7 for $U>0$.

Our derivation is only valid in the absence of the Rashba spin-orbit coupling $\alpha$. However, the numerical results in Fig. 3 show that the low-temperature Curie law in $\chi_{s}$ is the same also for $\alpha \neq 0$.

\section{APPENDIX B: SPIN SUSCEPTIBILITY AND GROUND-STATE DEGENERACY FOR FOUR $\pi$ FLUXES}

The results for the Kane-Mele-Hubbard model with four $\pi$ fluxes shown in Fig. 8 reveal a $\frac{2}{k_{B} T}$ Curie law at low temperatures and a $\frac{4}{k_{B} T}$ Curie law at higher temperatures. This finding can be understood as corresponding to either two or four noninteracting spins. The latter case corresponds to the spatially separate spin fluxon and an effective spin-1/2 Kramers doublet (formed by the three nearby spin fluxons) in the regime where $\chi_{s} \approx \frac{2}{k_{B} T}$ and to four noninteracting spin fluxons in the regime where $\chi_{s} \approx \frac{4}{k_{B} T}$.

The cluster formed by the three nearby spin fluxons has the possible configurations 


$$
\begin{aligned}
& \left|M^{z}\right|=\frac{3}{2}:\{|\uparrow \uparrow \uparrow\rangle,|\downarrow \downarrow \downarrow\rangle\}, \\
& \left|M^{z}\right|=\frac{1}{2}:\{|\uparrow \downarrow \downarrow\rangle,|\downarrow \uparrow \downarrow\rangle,|\downarrow \downarrow \uparrow\rangle,|\downarrow \uparrow \uparrow\rangle,|\uparrow \downarrow \uparrow\rangle,|\uparrow \uparrow \downarrow\rangle,
\end{aligned}
$$

where $M^{z}$ denotes the total spin in the $z$ direction. Since the exciton-mediated interaction in the Kane-Mele-Hubbard model has the form given in Eq. (7), and hence promotes spin flips, the ground state can be expected to have $\left|M^{z}\right|=$ $1 / 2$. The above-mentioned effective spin- $1 / 2$ doublet then corresponds to the two possible values $M^{z}= \pm 1 / 2$.

The degeneracy of the ground state depends on the sign of the interaction. Considering $M^{z}=1 / 2$, we have the allowed states $|\downarrow \uparrow \uparrow\rangle,|\uparrow \downarrow \uparrow\rangle$, and $|\uparrow \uparrow \downarrow\rangle$. The spin-flip terms that connect these states are of the form $J\left(S_{i+1}^{+} S_{i}^{-}+S_{i}^{+} S_{i+1}^{-}\right)$, with periodic boundary conditions. An equivalent representation is given by the Hamiltonian

$$
H=J \sum_{j}(|j+1\rangle\langle j|+| j\rangle\langle j+1|),
$$

which describes the hopping of a particle (the spin-down) on a three-site ring, with $|1\rangle=|\downarrow \uparrow \uparrow\rangle$, etc. The eigenstates are obtained by Fourier transformation and have the form

$$
|k\rangle=\frac{1}{\sqrt{3}} \sum_{j=1}^{3} e^{\mathrm{i} k j}|j\rangle, \quad k=0, \pm \frac{2 \pi}{3} .
$$

The eigenvalues are given by

$$
E(k)=2 J \cos k .
$$

For $J<0$, the ground state has $k=0$ and energy equal to $2 J$. For $J>0$, the ground state is chiral, with $k= \pm 2 \pi / 3$ and energy equal to $-J$. Taking into account the sector $M^{z}=-1 / 2$, we find a total ground-state degeneracy of two in the ferromagnetic case $(J<0)$ and four in the antiferromagnetic case $(J>0)$.

\section{APPENDIX C: FOURIER TRANSFORM OF THE EXCITON PROPAGATOR}

The exciton propagator in Eq. (7) takes the form

$$
D(\boldsymbol{q}, \tau)=\frac{e^{\tau \omega(\boldsymbol{q})}}{e^{\beta \omega(\boldsymbol{q})}-1}-\frac{e^{-\tau \omega(\boldsymbol{q})}}{e^{-\beta \omega(\boldsymbol{q})}-1},
$$

with the exciton energy

$$
\omega(\boldsymbol{q})=\sqrt{v^{2}|\boldsymbol{q}-\boldsymbol{Q}|^{2}+\Delta_{s}^{2}} .
$$

In the low-temperature limit $\beta \rightarrow \infty$, the propagator becomes $D(\boldsymbol{q}, \tau)=e^{-\tau \omega(\boldsymbol{q})}$. Setting $\boldsymbol{q}^{\prime}=\boldsymbol{q}-\boldsymbol{Q}$, the Fourier transform is given by

$$
D(\boldsymbol{r}, \tau)=e^{\mathrm{i} \boldsymbol{Q} \cdot \boldsymbol{r}} \int \mathrm{d}^{2} q^{\prime} e^{\mathrm{i} \boldsymbol{q}^{\prime} \cdot \boldsymbol{r}} e^{-\omega\left(\boldsymbol{q}^{\prime}+\boldsymbol{Q}\right) \tau} .
$$

Assuming $\Delta_{s} \gg v$, we can expand to obtain

$$
\omega\left(\boldsymbol{q}^{\prime}+\boldsymbol{Q}\right) \approx \Delta_{s}\left[1+\frac{\boldsymbol{v}^{2}\left|\boldsymbol{q}^{\prime}\right|^{2}}{2 \Delta_{s}^{2}}\right]
$$

Taking the continuum limit, the Fourier transform involves the product of two Gaussian integrals, and the result is given by Eq. (8).

[1] J.E. Moore, The Birth of Topological Insulators, Nature (London) 464, 194 (2010).

[2] M.Z. Hasan and C.L. Kane, Colloquium: Topological Insulators, Rev. Mod. Phys. 82, 3045 (2010).

[3] C. L. Kane and E. J. Mele, $Z_{2}$ Topological Order and the Quantum Spin Hall Effect, Phys. Rev. Lett. 95, 146802 (2005).

[4] Andreas P. Schnyder, Shinsei Ryu, Akira Furusaki, and Andreas W.W. Ludwig, Classification of Topological Insulators and Superconductors in Three Spatial Dimensions, Phys. Rev. B 78, 195125 (2008).

[5] C.L. Kane and E. J. Mele, Quantum Spin Hall Effect in Graphene, Phys. Rev. Lett. 95, 226801 (2005).

[6] Congjun Wu, B. Andrei Bernevig, and Shou-Cheng Zhang, Helical Liquid and the Edge of Quantum Spin Hall Systems, Phys. Rev. Lett. 96, 106401 (2006).

[7] Cenke Xu and J.E. Moore, Stability of the Quantum Spin Hall Effect: Effects of Interactions, Disorder, and $\mathrm{Z}_{2}$ Topology, Phys. Rev. B 73, 045322 (2006).

[8] B. Andrei Bernevig, Taylor L. Hughes, and Shou-Cheng Zhang, Quantum Spin Hall Effect and Topological Phase Transition in HgTe Quantum Wells, Science 314, 1757 (2006).

[9] Markus König, Steffen Wiedmann, Christoph Brüne, Andreas Roth, Hartmut Buhmann, Laurens W. Molenkamp, Xiao-Liang Qi, and Shou-Cheng Zhang, Quantum Spin Hall Insulator State in HgTe Quantum Wells, Science 318, 766 (2007).

[10] M. Hohenadler and F. F. Assaad, Correlation Effects in Two-Dimensional Topological Insulators [J. Phys. Condens. Matter (to be published)].

[11] Chang-Yu Hou, Claudio Chamon, and Christopher Mudry, Electron Fractionalization in Two-Dimensional Graphenelike Structures, Phys. Rev. Lett. 98, 186809 (2007).

[12] Ying Ran, Ashvin Vishwanath, and Dung-Hai Lee, SpinCharge Separated Solitons in a Topological Band Insulator, Phys. Rev. Lett. 101, 086801 (2008).

[13] Andreas Ruegg and Gregory A. Fiete, Topological Order and Semions in a Strongly Correlated Quantum Spin Hall Insulator, Phys. Rev. Lett. 108, 046401 (2012).

[14] G. A. Fiete, V. Chua, X. Hu, M. Kargarian, R. Lundgren, A. Ruegg, J. Wen, and V. Zyuzin, Topological Insulators and Quantum Spin Liquids, Physica (Amsterdam) 44, 845 (2012).

[15] Z. Y. Meng, T.C. Lang, S. Wessel, F. F. Assaad, and A. Muramatsu, Quantum Spin-Liquid Emerging in TwoDimensional Correlated Dirac Fermions, Nature (London) 464, 847 (2010). 
[16] M. Hohenadler, T. C. Lang, and F. F. Assaad, Correlation Effects in Quantum Spin-Hall Insulators: A Quantum Monte Carlo Study, Phys. Rev. Lett. 106, 100403 (2011).

[17] S. Raghu, Xiao-Liang Qi, C. Honerkamp, and ShouCheng Zhang, Topological Mott Insulators, Phys. Rev. Lett. 100, 156401 (2008).

[18] Dmytro Pesin and Leon Balents, Mott Physics and Band Topology in Materials with Strong Spin-Orbit Interaction, Nat. Phys. 6, 376 (2010).

[19] B. Beri and N. R. Cooper, $Z_{2}$ Topological Insulators in Ultracold Atomic Gases, Phys. Rev. Lett. 107, 145301 (2011).

[20] K. Sun, W. V. Liu, A. Hemmerich, and S. Das Sarma, Topological Semimetal in a Fermionic Optical Lattice, Nat. Phys. 8, 67 (2011).

[21] Qian Niu, D. J. Thouless, and Yong-Shi Wu, Quantized Hall Conductance as a Topological Invariant, Phys. Rev. B 31, 3372 (1985).

[22] Zhong Wang, Xiao-Liang Qi, and Shou-Cheng Zhang, Topological Order Parameters for Interacting Topological Insulators, Phys. Rev. Lett. 105, 256803 (2010).

[23] V. Gurarie, Single-Particle Green's Functions and Interacting Topological Insulators, Phys. Rev. B 83, 085426 (2011).

[24] Zhong Wang, Xiao-Liang Qi, and Shou-Cheng Zhang, Topological Invariants for Interacting Topological Insulators with Inversion Symmetry, Phys. Rev. B 85, 165126 (2012).

[25] Zhong Wang and Shou-Cheng Zhang, Simplfied Topological Invariants for Interacting Insulators, Phys. Rev. X 2, 031008 (2012).

[26] Tsuneya Yoshida, Satoshi Fujimoto, and Norio Kawakami, Correlation Effects on a Topological Insulator at Finite Temperatures, Phys. Rev. B 85, 125113 (2012).

[27] Jan Carl Budich, Ronny Thomale, Gang Li, Manuel Laubach, and Shou-Cheng Zhang, Fluctuation-Induced Topological Quantum Phase Transitions in Quantum Spin-Hall and Anomalous-Hall Insulators, Phys. Rev. B 86, 201407 (2012).

[28] Christopher N. Varney, Kai Sun, Marcos Rigol, and Victor Galitski, Topological Phase Transitions for Interacting Finite Systems, Phys. Rev. B 84, 241105 (2011).

[29] Y. Ran, Y. Zang, and A. Vishwanath, One-Dimensional Topologically Protected Modes in Topological Insulators with Lattice Dislocations, Nat. Phys. 5, 298 (2009).

[30] V. Juricic, A. Mesaros, R.-J. Slager, and J. Zaanen, Universal Probes of Two-Dimensional Topological Insulators: Dislocation and $\pi$ Flux, Phys. Rev. Lett. 108, 106403 (2012).

[31] Xiao-Liang Qi and Shou-Cheng Zhang, Spin-Charge Separation in the Quantum Spin Hall State, Phys. Rev. Lett. 101, 086802 (2008).

[32] G. Rosenberg, H.-M. Guo, and M. Franz, Wormhole Effect in a Strong Topological Insulator, Phys. Rev. B 82, 041104 (2010).

[33] F. D. M. Haldane, Model for a Quantum Hall Effect without Landau Levels: Condensed-Matter Realization of the Parity Anomaly, Phys. Rev. Lett. 61, 2015 (1988).
[34] A. Shitade, H. Katsura, J. Kunes, X.-L. Qi, S.-C. Zhang, and N. Nagaosa, Quantum Spin Hall Effect in a Transition Metal Oxide $\mathrm{Na}_{2} \mathrm{IrO}_{3}$, Phys. Rev. Lett. 102, 256403 (2009).

[35] A. Singha, M. Gibertini, B. Karmakar, S. Yuan, M. Polini, G. Vignale, M. I. Katsnelson, A. Pinczuk, L. N. Pfeier, K. W. West, and V. Pellegrini, Two-Dimensional MottHubbard Electrons in an Artificial Honeycomb Lattice, Science 332, 1176 (2011).

[36] Conan Weeks, Jun Hu, Jason Alicea, Marcel Franz, and Ruqian Wu, Engineering a Robust Quantum Spin Hall State in Graphene via Adatom Deposition, Phys. Rev. X 1, 021001 (2011).

[37] L. Tarruell, D. Greif, T. Uehlinger, G. Jotzu, and T. Esslinger, Creating, Moving and Merging Dirac Points with a Fermi Gas in a Tunable Honeycomb Lattice, Nature (London) 483, 302 (2012).

[38] Y. A. Bychkov and E. I. Rashba Oscillatory Effects and the Magnetic Susceptibility of Carriers in Inversion Layers, J. Phys. C 17, 6039 (1984).

[39] A.H. Castro Neto, F. Guinea, N.M. R. Peres, K. S. Novoselov, and A. K. Geim, The Electronic Properties of Graphene, Rev. Mod. Phys. 81, 109 (2009).

[40] D. N. Sheng, Z. Y. Weng, L. Sheng, and F. D. M. Haldane, Quantum Spin-Hall Effect and Topologically Invariant Chern Numbers, Phys. Rev. Lett. 97, 036808 (2006).

[41] J. Hubbard, Electron Correlations in Narrow Energy Bands, Proc. R. Soc. A 276, 238 (1963).

[42] Stephan Rachel and Karyn Le Hur, Topological Insulators and Mott Physics from the Hubbard Interaction, Phys. Rev. B 82, 075106 (2010).

[43] Dong Zheng, Guang-Ming Zhang, and Congjun Wu, Particle-Hole Symmetry and Interaction Effects in the Kane-Mele-Hubbard Model, Phys. Rev. B 84, 205121 (2011).

[44] C. Griset and C. Xu, Phase Diagram of the Kane-MeleHubbard Model, Phys. Rev. B 85, 045123 (2012).

[45] S.-L. Yu, X.C. Xie, and J.-X. Li, Mott Physics and Topological Phase Transition in Correlated Dirac Fermions, Phys. Rev. Lett. 107, 010401 (2011).

[46] Wei Wu, Stephan Rachel, Wu-Ming Liu, and Karyn Le Hur, Quantum Spin Hall Insulators with Interactions and Lattice Anisotropy, Phys. Rev. B 85, 205102 (2012).

[47] Dung-Hai Lee, Effects of Interaction on Quantum Spin Hall Insulators, Phys. Rev. Lett. 107, 166806 (2011).

[48] M. Hohenadler, Z. Y. Meng, T. C. Lang, S. Wessel, A. Muramatsu, and F. F. Assaad, Quantum Phase Transitions in the Kane-Mele-Hubbard Model, Phys. Rev. B 85, 115132 (2012).

[49] J.E. Hirsch, D. J. Scalapino, R. L. Sugar, and R. Blankenbecler, Monte Carlo Simulations of OneDimensional Fermion Systems, Phys. Rev. B 26, 5033 (1982).

[50] F. F. Assaad and H. G. Evertz, in Computational Many Particle Physics, edited by H. Fehske, R. Schneider, and A. Weie, Lecture Notes in Physics Vol. 739 (Springer Verlag, Berlin, 2008), p. 277.

[51] W. P. Su, J. R. Schrieer, and A.J. Heeger, Solitons in Polyacetylene, Phys. Rev. Lett. 42, 1698 (1979).

[52] Massimo Campostrini, Martin Hasenbusch, Andrea Pelissetto, Paolo Rossi, and Ettore Vicari, Critical 
Behavior of the Three-Dimensional XY Universality Class, Phys. Rev. B 63, 214503 (2001).

[53] L. Gaudreau, G. Granger, A. Kam, G. C. Aers, S. A. Studenikin, P. Zawadzki, M. Pioro-Ladriere, Z. R. Wasilewski, and A.S. Sachrajda, Coherent Control of Three-Spin States in a Triple Quantum Dot, Nat. Phys. 8, 54 (2011).

[54] A. Izmalkov, M. Grajcar, S.H.W. van der Ploeg, U. Hubner, E. Il'ichev, H.-G. Meyer, and A. M. Zagoskin, Measurement of the Ground-State Flux Diagram of Three Coupled Qubits as a First Step Towards the Demonstration of Adiabatic Quantum Computation, Europhys. Lett. 76, 533 (2006).

[55] A. F. Albuquerque, F. Alet, P. Corboz, P. Dayal, A. Feiguin, S. Fuchs, L. Gamper, E. Gull, S. Gurtler, A. Honecker, R. Igarashi, M. Korner, A. Kozhevnikov, A. Lauchli, S. R. Manmana, M. Matsumoto, I.P. McCullochc, F. Michel, R. M. Noack, G. Pawlowski, L. Pollet, T. Pruschke, U. Schollwock, S. Todo, S.
Trebst, M. Troyer, P. Werner, and S. Wessel for the ALPS Collaboration, The ALPS Project Release 1.3: Open-Source Software for Strongly Correlated Systems, J. Magn. Magn. Mater. 310, 1187 (2007).

[56] M. J. Bhaseen, A. O. Silver, M. Hohenadler, and B.D. Simons, Feshbach Resonance in Optical Lattices and the Quantum Ising Model, Phys. Rev. Lett. 103, 265302 (2009).

[57] K. K. Gomes, W. Mar, W. Ko, F. Guinea, and H. C. Manoharan, Designer Dirac Fermions and Topological Phases in Molecular Graphene, Nature (London) 483, 306 (2012).

[58] N. Goldman, I. Satija, P. Nikolic, A. Bermudez, M. A. Martin-Delgado, M. Lewenstein, and I. B. Spielman, Realistic Time-Reversal Invariant Topological Insulators with Neutral Atoms, Phys. Rev. Lett. 105, 255302 (2010).

[59] A. Rüegg and C. Lin, Bound States of Conical Singularities in Graphene-Based Topological Insulators, Phys. Rev. Lett. 110, 046401 (2013). 